\title{
Australie : Les droits d'inscription au cœur de la réforme de l'enseignement supérieur
}

Le point sur...

Marion Latour

(2) OpenEdition

Journals

Édition électronique

URL : http://journals.openedition.org/ries/1614

DOI : 10.4000/ries. 1614

ISSN : 2261-4265

Éditeur

Centre international d'études pédagogiques

Édition imprimée

Date de publication : 1 septembre 2003

Pagination : 20-22

ISBN : 978-2-85420-557-1

ISSN : $1254-4590$

Référence électronique

Marion Latour, «Australie : Les droits d'inscription au cœur de la réforme de l'enseignement supérieur », Revue internationale d'éducation de Sèvres [En ligne], 33 | septembre 2003, mis en ligne le 12 avril 2012, consulté le 20 avril 2019. URL : http://journals.openedition.org/ries/1614 ; DOI : 10.4000/ ries. 1614

Ce document a été généré automatiquement le 20 avril 2019.

(c) Tous droits réservés 


\title{
Australie : Les droits d'inscription au cour de la réforme de l'enseignement supérieur
}

Le point sur...

\author{
Marion Latour
}

1 Le 18 février 2003, le gouvernement australien a approuvé le projet de réforme de l'enseignement supérieur présenté par Brendan Nelson, actuel ministre de l'Éducation, des sciences et de la formation. L'objectif premier de la réforme est de donner une plus grande souplesse aux trente huit universités publiques que compte le pays. Les mots clés de la réforme sont capacité financière, qualité, égalité et diversité. Le ministre a organisé cinq mois de consultation en 2002 auprès des principaux acteurs de l'enseignement supérieur, des enseignants aux entreprises en passant par la minorité aborigène ${ }^{1}$. L'aspect financier a monopolisé les discussions sur la réforme. Cette question s'est de nouveau trouvée au cœur des débats au moment du vote du budget, à la mi-mai 2003.

Depuis les années soixante-dix, le gouvernement fédéral joue un rôle croissant dans l'éducation. Les incitations à la réforme et l'engagement financier du gouvernement ont orienté l'éducation vers une approche plus nationale. En 2001, près de $44 \%$ du budget des institutions du supérieur provenait du gouvernement fédéral, contre 1,7 \% des États. Les étudiants en cours de scolarité ont contribué à hauteur de $20 \%$ par les droits d'inscription et les anciens étudiants bénéficiaires du Higher Educational Contribution Scheme à hauteur de $17,4 \%{ }^{2}$ par prélèvement supplémentaire du revenu imposable. Introduit en 1989 par le précédent gouvernement, ce système d'emprunt permet aux étudiants de retarder leur remboursement jusqu'au moment où ils en ont les moyens. Réservé aux seules institutions publiques ${ }^{3}$, il a contribué à l'accroissement du nombre d'étudiants et a constitué la principale source de crédits supplémentaires, nécessaires au système éducatif pour se développer. Non seulement M. Nelson est favorable au HECS mais il a en 2002 introduit le Postgraduate Education Loans Scheme pour les étudiants de troisième cycle. Il s'agit également de reporter le paiement mais, à la différence du HECS, 
ce sont les universités qui fixent les droits d'entrée. D'autre part le gouvernement verse le montant du prêt directement à l'institution et non à l'étudiant. L'endettement des étudiants est estimé à 11 milliards de dollars australiens ${ }^{4}$ d'ici à 2005. De fait, $80 \%$ d'entre eux travaillent parallèlement à leurs études, dont bon nombre pour subvenir à leurs besoins élémentaires ${ }^{5}$.

En juillet 2002, le ministère a publié un ouvrage qui fait la synthèse des différentes consultations sur le thème du financement, Setting firm foundations: financing Australian higher education ${ }^{6}$. La dérégulation des droits d'inscription y est envisagée parallèlement au relèvement du seuil du revenu à partir duquel les diplômés commencent à rembourser leur emprunt, ainsi qu'à une augmentation des prêts accordés aux étudiants les plus défavorisés. Ce rapport envisage également de diversifier les sources de financement des universités, notamment en développant encore d'avantage l'accueil des étudiants étrangers ${ }^{7}$, en exemptant les universités de certains impôts et en incitant fiscalement les entreprises à investir dans les universités. Or un rapport de Price Waterhouse Coopers ${ }^{8}$ révèle l'ampleur des préjugés existant entre le monde universitaire et les milieux d'affaires. Globalement, les entreprises ne voient pas quel parti elles peuvent tirer d'une collaboration plus étroite avec les universités.

Dans la continuité du rapport de juillet 2002, M. Nelson a déendu les points suivants lors de la présentation du budget fédéral :

- 15,8 milliards de dollars prévus pour l'ensemble du secteur éducatif en 2003-2004, soit un budget en augmentation ${ }^{9}$;

- une enveloppe supplémentaire de 1,46 milliard de dollars investis sur cinq ans dans le supérieur ;

- doublement des places réservées aux étudiants payant leurs études à taux plein qui pourront en outre emprunter dans la limite de 50000 dollars (ce plafond n'étant pas appliqué aux cursus longs) ;

- relèvement du seuil salarial à partir duquel les diplômés doivent rembourser leurs études (passage de 24365 à 30000 dollars en 2005-2006);

- allocation de bourses en premier cycle universitaire réservé aux milieux socio-économiques faibles, aux zones rurales et éloignées et aux étudiants indigènes, soit 160,2 milliards de dollars sur quatre ans,

- augmentation de plus de $20 \%$ d'ici à 2005 des droits d'inscription pour le premier cycle universitaire,

- liberté laissée aux universités d'augmenter les droits d'inscription, dans des limites fixées, pour les places subventionnées par le gouvernement,

- dispositions de remboursement du HECS légèrement moins favorables pour les étudiants qui s'acquittent immédiatement de leur remboursement.

5 Si les premiers points recueillent l'approbation d'une majorité, les derniers font l'objet de vifs débats. Le ministre doit affronter le mécontentement des étudiants, très opposés à l'augmentation des droits d'inscription. Le syndicat des étudiants, the National Union of Students, menace de manifester massivement en août et de lancer une campagne dans tout le pays pour obtenir des alliances avec les partis minoritaires. Les travaillistes et les démocrates, également hostiles à la réforme, demandent une enquête sénatoriale. En revanche, les présidents d'université se sont déclarés favorables à une dérégulation partielle des droits d'inscription. De son côté, le comité des présidents d'universités, the Australian Vice-Chancellors Committee, préconise d'une part la contractualisation des enseignants, d'autre part la corrélation entre les subventions versées et les résultats 
obtenus par les universités. Il désire également que le seuil salarial de remboursement du prêt HECS soit remonté à 35000 dollars ou s'ajuste au salaire moyen des jeunes diplômés débutants. Si le comité parle d'une seule voix, des différences d'appréciation se font entendre en réalité. Les universités les plus anciennes, également les plus établies, risquent de profiter davantage de la hausse des frais de scolarité ; or leur influence est disproportionnée par rapport au nombre de leurs étudiants.

Alors que Brendan Nelson était bien décidé à faire passer sa réforme intégralement, il a donné le 23 mai 2003 un premier signe de compromis en se déclarant prêt à augmenter le plafond de l'emprunt fixé à 50000 dollars annuels pour les étudiants à taux plein. Parallèlement, les responsables du ministère organisent des rencontres dans tous les États pour expliquer les nouvelles dispositions. Il est cependant trop tôt pour que les universités se fassent une idée précise de leurs conséquences. En juillet se tiendra la rencontre de l'ensemble des ministres de l'éducation. Ils ne manqueront pas de débattre d'une autre question épineuse : la distribution des 25000 places entièrement subventionnées, que le gouvernement propose pour pallier les inscriptions en surnombre. En effet, face au déficit de places, les universités adoptent deux attitudes. Certaines acceptent plus d'inscrits qu'elles n'en ont la capacité afin de recueillir des fonds supplémentaires par le biais des droits d'inscription; à l'inverse, d'autres refusent des inscriptions. On estime que la demande non satisfaite se serait accrue de $60 \%$ depuis 2001. À la rentrée 2003, c'est entre 18700 et 25700 candidats qui, bien qu'éligibles, se sont vu refuser l'entrée à l'université, soit l'équivalent d'une université de taille moyenne

\section{NOTES}

1. De fin avril 2002 à septembre, sept rapports de propositions de réforme ont été discutés lors de 49 forums et plus de 730 avis ont été recueillis. Le forum qui s'est tenu au Parlement les 3 et 4 octobre a été le point d'orgue de la consultation. Il a permis de formaliser les cinq mois de débat.

2. Consulter sur le site du ministère le document intitulé Finance 2001 : selected higher education statistics http://www.dest.gov.au/highered/statistics/finance/2001/finance_2001_final.pdf

3. Autorisé également pour l'Open Learning Australian Consortium. Ceci crée une inégalité vis à vis des étudiants inscrits dans des établissements privés, dont les cours sont pourtant reconnus par l'État fédéral.

4. Soit 6,71 milliards d'euros.

5. Selon l'association Australian scolarships group les étudiants doivent payer entre 54000 et 130000 dollars pour obtenir leur diplôme (soit entre 32926 et 79267 euros). Fondé sur 30 universités, ce rapport prend en compte les frais de scolarité, le logement, le transport, les livres, les charges exceptionnelles, la nourriture, les vêtements et un budget loisir de 30 dollars par semaine.

6. http://www.backingaustraliasfuture.gov.au/publications/setting_firm_foundations/ default.htm

7. Il est estimé que les étudiants étrangers génèrent plus de 4 milliards de dollars par an. Dans son budget, M. Nelson a prévu 113 millions de dollars sur 4 ans pour favoriser leur promotion, chiffre que l'on peut rapprocher des 123 millions prévus pour soutenir les universités régionales. 
8.

http://www.pwcglobal.com/Extweb/

indissue.nsf/2e7e9636c6b92859852565e00073d2fd/94cab7f05fb6bafaca256c86000dfa43/\$FILE/

Higher_Education_Review_2002.pdf

9. En 2002-2003 le budget s'élevait à 14,7 milliards de dollars, soit une hausse de $7 \%$ par rapport à 2001-2002.

INDEX

Index géographique : Australie

Mots-clés : enseignement supérieur, droit d'inscription

\section{AUTEUR}

\section{MARION LATOUR}

Documentaliste, centre de ressources documentaires du CIEP. 